\title{
Perspectives on Counselor Education Collaborative Scholarship
}

\author{
Michael Brooks ${ }^{1}$, Jeff Wolfgang ${ }^{2}$, Justin Adams ${ }^{2}$, Kim Lee Hughes ${ }^{3}$ \\ ${ }^{1}$ Associate Professor, North Carolina A\&T State University, United States \\ ${ }^{2}$ Assistant Professor, North Carolina A\&T State University, United States \\ ${ }^{3}$ Assistant Professor, Clark-Atlanta University, United States \\ Correspondence: Michael Brooks, Associate Professor, North Carolina A\&T State University, United States.
}

Received: December 11, 2020

Accepted: February 16, $2021 \quad$ Available online: February 23, 2021

doi:10.11114/ijsss.v9i2.5101

URL: https://doi.org/10.11114/ijsss.v9i2.5101

\begin{abstract}
A collective case study employs a multiple-case design to investigate engaging, collaborative academic writing methods in counselor education. The researchers were particularly interested in understanding successful collaborative counseling research practices centered around Harshada Patel CoSpaces Collaborative Working Model (CCWM). Analysis from two rounds of interviews with 11 participants revealed the central tenets needed to complete scholarly work in Counselor Education. The researchers discovered leadership, institutional capacity, and communication as the three major categories based on the transcription content analysis. According to the results, counselor educators can embrace collaboration into their research practice and identity as it is a natural extension of humanistic philosophy.
\end{abstract}

Keywords: academia collaboration, counselor education scholarship, research capacity

\section{Introduction}

The counseling profession has evolved and taken many critical initiatives to advance a robust, professional identity (Woo et al., 2017). An essential aspect of our counseling professional identity is expanding and promoting counseling research (Kaplan \& Gladding, 2011). The relational orientation of counseling is central to developing collaborative research in counselor education (Parrow et al., 2019). Counselor education researchers may benefit from exploration in effective collaboration scholarship practices. Empirical Collaborative research efforts could have particular importance in assessing, measuring, and evaluating effective counseling practices.

Over the past three decades, US colleges and universities have increased their focus on research outcomes and productivity as metrics for promotion and tenure (Allen, Huggins-Hoyt, Holosko, \& Briggs, 2017). Thus, academia is becoming more competitive, and the stakes are higher in an often publish or perish environment. As Allen et al. (2017) suggest, this publishing pressure finds faculty in a delicate balancing act of finding time for the scholarship when they often have solemn teaching and service loads. Research on co-authoring increases in the humanities (Lunsford and Ede 1990; Forman 1992) and Computer Supported Cooperative Work (CSCW; Patel, Pettitt, \& Wilson, 2012). An increasing number of interview studies (e.g., Posner 1991; Rimmershaw 1992) and surveys (e.g., Lunsford and Ede 1990; Couture and Rymer 1991; Chandler 1992; Beck 1993) are becoming available. Few longitudinal studies on the process of collaborative writing exist; however, self-reports of the knowledge of co-authoring are growing in numbers.

\subsection{What is Collaborative Scholarship}

Collaborative scholarship, confirmed or open, emerged from learners' association, from a rational population whose branches help as knowledge and research partners. Austin and Baldwin (1992) also described faculty collaboration as a mutual effort including united goals, effort, and products. These definitions are broad and expansive because university scholarship changes depending on the purpose and the contributors' specific information and place on the career ladder. All to say, collaborative research is a collegial experience leading to the making of new knowledge. Regardless of the collaboration, professional development, or personal growth, the collective intends to synthesize interests to inform knowledge generation and promote faculty development.

Collaborative scholarship is practical and adept. Collaborative scholarship can promote teamwork, increase faculty productivity, and stimulate more profound investigation of different dimensions of the same phenomenon, thus producing more information about that aspect, keeping faculty driven, plus enriching an individuals' points of view and expertise 
(Austin \& Baldwin, 1992). If associates begin a project together, intellectual stimulation is generated (Young, 1984). As a form of professional development, partnerships can enhance each member with a novel view on otherwise common concepts.

Research is becoming increasingly collaborative; driving collaborative research is creating best practices (Leahey, 2016). It is possible that as the counseling profession increases its specialty areas, our need for collaborative research will continue to grow (Woo \& Henfield, 2015). Collaboration appears to drive the development and dissemination of evidence-based practices in areas centered around practical assessment, measurement, and evaluation of effective counseling practices (David, Lynn, \& Montgomery, 2018).

\subsection{Review of Literature}

While exploring the literature in counseling settings, there have been multiple studies conducted explaining the process of how counselors collaborate to produce successful outcomes for their clients (Katz \& Martin, 1997; Holstein, James, \& Gubrium, 2012, \& Brooks et al., 2020). With this notion of collaboration as fundamental in the profession, this study's researchers were surprised to find limited resources that speak to scholarly research and collaborative efforts. Scouring through counseling journals; however, over half of the journals accepted have three or more authors (Katz \& Martin, 1997; James \& Gubrium, 2012). Collaboration is happening, but the effective strategies these researchers used to ensure a successful outcome is unsure. The current inquiry is an exploration of possible effective practices in scholarship/research collaborations for counselor educators.

Effective collaboration in counseling scholarship should mirror the theoretical foundation embodied by the counseling profession. Articles by Bozeman et al. (2015) and Patel et al. (2012) provided provisional models to guide our exploration of effective collaboration in counseling scholarship/research. They suggested responses and solutions to increasingly competitive work settings were to develop collaboration efforts. Patel et al. (2012) identified seven potential factors involved in collaboration: context, support, tasks, interaction process, teams, individuals, and overarching factors. Bozeman et al. (2015) identified three factors that may describe collaboration dynamics: external (discipline characteristics, commerce), collaborator (work-style fit, career stage and motives, and gender), and team management factors (collaboration management structures).

Collaborative work is known to add zest to the research process (Ayoola et al., 2017; Katz \& Martin, 1997). Moreover, the writing process can be lonely and isolating (Tsatsou, 2016). When scholars come together to produce scholarship and research, they get to share meaningful experiences. The researchers were interested in closed' co-authoring instead of, for example, editing. Closed co-authoring is the crafting of manuscripts by persons, where the names appearing in the author list are those of people who were involved in the development of the document (McConnell, 2016). A particular format and method for effective collaborations might help implement a co-authored group, especially those working together across geographic distances.

The development of communication technology has made it easier and more cost-effective for professionals to communicate with colleagues over a wider area than ever before (Bozeman \& Youtie, 2016). Some find it convenient or necessary to work with great distances away and collaborate on documents with them. The chances of increasing co-authors are distributed over a wide range and must use telecommunications technology to support communication and writing activity (Austin et al., 2012). Co-authoring can be defined in many ways (Lunsford and Ede 1990), giving different perspectives on co-authoring practices (Couture and Rymer, 1991). The assigning of authorship to academic papers is a study (e.g., Trimbur and Braun 1992).

There is a rich history that speaks to collaboration; researchers have conducted multiple ethnographies and case studies of workplace collaboration (Blakeslee, 1993; Cross, 1990, 1993, 1994, 2001; Dautermann, 1993, 1997; Doheny-Farina, 1986; Locker, 1992; Weber, 1991; Winsor, 1989). These studies focused on co-drafting and activities that lead to published documents. Jason Palmeri (2004) chose to create a collaborative theory for those attempting to practice effective collaboration for project success. The four areas of his theory are (1) discourse communities, (2) addressing collaborative conflicts, (3) narrative dialogues, and (4) collaborative practices. Implications of utilizing an actual "theory" to guide collaboration resulted in positives of maximized outcomes, diverse teams, and efficient timelines. Exploring this collaborative theory was adopted and practiced in many other disciplines not related to counselor education or academia.

Professional disciplines such as business, nursing, medical, and law have long practiced and developed methods for best practices in effective collaboration (Couture \& Rymer, 1989; Ede \& Lunsford, 1990; Faigley \& Miller, 1982; Belanger \& Greer, 1992; Burnett, 1993; Forman, 1991; Rogers \& Horton, 1992). Albeit, there is little to no evidence of significant collaboration within the counseling profession of higher academia. Questions arise as to why helping professions such as nursing and medical fields offer a standard of effective collaboration for scholarship, but counseling (which is considered a helping profession) does not (World Health Organization, 2010). 
Therefore, it is clear from previous literature (Driscoll et al., 2009; Paetow et al., 2018) there is a need to present a research study on effective collaboration that speaks specifically to counselor educators. Moreover, following a significant process on co-authorship groups, working across geographic distances utilizing a format and method can yield successful scholarship opportunities. This study explores using the model forms of collaboration in higher education to extend counseling research/ scholarship.

Based on a comprehensive search using online and library sources, no counselor education literature spoke to collaborating successfully. Our investigations utilized the universities OneSearch, which aggregates most of the library's web-based databases and print resources covering Academic search complete, Worldcat, ProQuest Central, and Wiley Online. These hold the bulk of ACA journals, many liberal arts and science journals, and technology journals. We also implemented a google scholar search, which resulted in no findings on how to write cooperatively.

According to the World Health Organization, "Interprofessional education occurs when students from two or more professions learn about, from, and with each other to enable effective collaboration and improve health outcomes" (World Health Organization, 2010, p.10). The Institute for Healthcare Improvement called on academic health institutions to implement training programs to prepare profesionals to work effectively together in collaborative practice environments. Overlapping mental health professions' education can be a pathway for quality improvement and impetus for our educational approach changes. It is mainly for this reason why we believe an empirical exploration of this topic is warranted.

As teamwork in scholarship/research increases, it is essential to establish acceptable practices in collaboration. Laying clear group rules (prenuptial agreement for a scientist) and having an open discussion about expectations can help the cooperation run smoothly. Coupled with counseling's collaborative framework, where common goals lead to success, it appears that an empirical study on cooperative counselor education would be beneficial and necessary. The aim of this current study was to investigate ways to engage in collaborative academic writing in counselor education. The researchers were particularly interested in understanding successful collaborative counseling research practices.

\section{Method}

As part of an investigation of counselor educators' academic collaborative writing experiences, we provide the principal findings of this collective case study exploration. A collective case study employs a multiple-case design to provide an in-depth, multi-faceted understanding of the phenomena while incorporating context into the inquiry(Crowe et al., 2011). In this study, the researchers selected a split panel of counseling research experts to gather their contextualized experiences. The context in our study will be the meaningful and dynamic interactions between the phenomenon (collaborative writing experiences of counselor educators) and the context (professional identity, relational orientation, and academic productivity, for example) that are currently not fully understood (Creswell \& Guetterman, 2019; Yin, 2018).

The multiple-case design was analyzed using a directed qualitative content analysis approach, as Brooks et al. (2020) exhibited. This culture-centered analysis used prior research to guide and structure the communication artifacts to determine critical concepts, patterns, and themes around collaborative writing practices in counselor education across the United States (Hsieh \& Shannon, 2005; Vaismoradi et al., 2016). Saldaña (2016) asserted this method is particularly appropriate for exploring intrapersonal and interpersonal meanings in communication. Embedded in this culture-centered approach was a positional and textual collaborative reflexivity that required the researchers to critically reflect and deconstruct the relationships between counseling educator participants and the textual artifacts describing the collective writing experiences (Agee et al., 2013).

We strengthened our findings' credibility through discursive questioning and collaborative dialogue that engaged researchers and participants in a dialectic exchange (Goldstein \& Gigernzer, 2002). Considering the clinical and relational experiences supports the reader's understanding of the participant's subjective experiences (Cunningham \& Carmichael, 2018). As a part of the credibility building, the researchers engaged in peer debriefing (bracketing) and member checking during the development of the research questions, interpretation of textual responses, and subsequent content analysis process. The researchers conducted this process of gathering participants, developing research questions, conducting interviews, analyzing data, and synthesizing results over a year, beginning in April 2019 and ending October 2020. This lengthy process aided in operationalizing patterns to produce a credible and valid presentation of the researchers' findings (Hsieh \& Shannon, 2005).

Bracketing is the ongoing process of suspending preconceptions, biases, and presuppositions through active reflection and dialogue (Tufford \& Newman, 2010). Member checking entailed sharing the final themes and patterns derived from the qualitative questionnaire to allow respondent validation (member checking). In member, checking participants were allowed to clarify and elaborate on the themes and patterns, at times providing thicker descriptions that dug deeper into their experiences (Creswell \& Guetterman, 2019). 
The research team was a multi-ethnic and mixed-gender group consisting of two African American males, one African American female, and a White male counselor educator at research-intensive universities. The authors carried their ethnocultural beliefs and actualized experiences of collaborative research in counselor education into this investigation. Researchers viewed, understood, and analyzed the from a constructivist, humanistic, and relational perspective compatible with the counseling profession (Vereen et al., 2014).

\subsection{Participants}

Creating an appropriate panel of experts is critical to the study's strength and validity (Clayton, 1997). Scheele (1975) suggested the forum develop a "contextual mapping that would describe the society's response to any complex issue" (p. 60). He also recommended that a split panel containing both practitioners and scholars may be of great benefit for a successful study.

A split panel of experts was composed of collaborative scholars and academic writing evaluators in the present study. To have been considered for the conference, potential participants have a successful and established track record of joint publications $(20+$ publications within the past five years) or served (within the past three years) as an editor of an academic counseling journal or editorial review board member.

The authors selected participants through the use of criterion-based purposive sampling. The authors gathered potential participants by compiling a list of flagship American Counseling Association's journal editors, editorial board members, and scholars most associated with counseling (verified by their vita, meeting the above criteria of $20+$ collaborative publications in the past five years). The authors used a non-probabilistic method based on the predetermined criteria (Patton, 2002). All of the editors were counselor educators who had full-time faculty appointments.

The authors contacted twenty potential participants via email, including seven editors/review members, seven collaborative scholars, and 13 individuals who met both criteria. Of the potential participants, 14 individuals indicated they would serve on the panel. Three panelists (s) withdrew from the study due to availability conflicts. The final panel consisted of 7 editorial board members, four scholars, for 11 participants that spanned across various United States of America locations.

\subsection{Measures}

The researchers created a list of questions based on the CoSpaces Collaborative Working Model (CCWM) Patel et al. (2012) and Bozeman et al. (2015) analysis of the CCWM model. However, the researchers did not reveal the theory or the study's specific purpose to the panel. The authors asked responsive questions based on four constructs (context, support, teams, and overarching factors). Context refers to circumstances which form the setting for an event, statement, or idea. Support, or the assistance given to enable functioning, consisted of tools, networks, resources, training, and team-building: teams or the coming together to achieve a common goal; the individual's needed and selecting them. Overarching factors necessary for several areas of the project consisted of trust, performance, and honesty. For our study, we were interested in determining how Counselor Educators can effectively collaborate in their research and scholarship writing from a panel of experts. The panelists responded to 9 semi-structured interview questions.

1. How is an environment which maximizes the abilities and willingness to foster scholarly collaborative relationships created?

2. As a collaborative leader, how are collaborators focused on short-term and medium-term goals?

3. How does your institution's organizational structure support scholarly collaboration?

4. How do you utilize networks in collaborative relationships?

5. What training has assisted you in facilitating collaborative research efforts?

6. Factors in Collaborative Success - What are the elements for scholarly collaboration success?

7. How do you (as lead) deal with the authorship order and the possibility of re-ordering the order?

8. If you (as lead author) have to remove a collaborative member, what are the circumstances?

9. How do you maintain collegial relationships while conducting/navigating "business?"

\subsection{Reflexivity and Trustworthiness}

In Round 1 of data collection, researchers sent the panelists an email outlining the study's procedures and instructions regarding how to access the initial open-ended questionnaire. Data was collected using Qualtrics, a web-based survey software package for the social sciences (qualtrics.com). In the first round of analysis, after the panelists' initial positions were identified. The panelists' responses were inputted into a codebook (excel doc) and organized by question items. The researchers implemented collaborative reflexivity by analyzing reactions in groups of 2 and 3 . Research colleagues 
discussed notes and analysis and compared agreements and disagreements for possible insights. The researchers used possible reflexivity discoveries to inform techniques going forward.

The researchers used data triangulation as participants answered multiple rounds of interview questions and follow-ups (Carter et al., 2014; Patton, 1999). To maintain this study's trustworthiness, the researchers decided to leave each reaction in its entirety, so researchers lost no information from both the overall essence of participants' answers and each message's nuances (Wilhelm, 2001).

During the second round of data collection, panelists received an email with instructions and a timeline for responding. This phase allowed panelists to evaluate all respondents' answers and reevaluate their own in light of the other represented points of view.

\section{Results}

The researchers were interested in understanding successful collaborative counseling research practices. Eleven counselor educators with strong histories as scholars were selected and interviewed. Their responses to questions regarding collaboration in counselor education were analyzed and reduced to the three previous state categories (leadership, institutional research capacity, communication). The results are in line with the literature (Vaismoradi, Jones, Turunen, et al., 2016, Patel, Pettitt, \& Wilson, J. R., 2012), in that these components increase the chances of research effort being successful when present.

\subsection{Leadership (Category 1)}

Consistent with the study results, leadership is vital in establishing collaborative scholarship (Amey \& Brown, 2005). Academic research leadership includes creating a vision, and setting up creative ideas, doing and providing teamwork. Influential leaders can influence people on research related behaviors, attitudes, and intellectual capacity. Some essential factors in predicting faculty productivity were early research socialization, working in a favorable environment, and assertive leadership (Thompson, Galbraith, \& Pedro, 2010). The lead author (often the research leader) has a concept. The sub-categories below were supported or related to academic research leadership.

\subsubsection{Soft Skills}

Under the leadership category, participants identified some particular areas, one being soft skills. The researchers collapsed the 2nd cycled themes into the sub-category, soft skills. Soft skills are the skills enabling one to fit in a place of work. Soft skills are generally one's personality, attitude, flexibility, motivation, and manners. In the application process, soft skills can be justification for employers keeping or promoting an employee. Soft skills are different from hard skills (also known as technical skills), directly relevant to the job. To succeed at collaborative writing, one must get along well with others, including co-authors, support staff, students, and anyone else you communicate with throughout the project. Below are the following themes (positive attitude, goal setting, and management).

Positive attitude. It is essential to embody an attitude of positivity, optimism, and enthusiasm. A positive attitude can do wonders in turning a project around - and it is contagious.

- Valuing all contributing authors Acknowledging/valuing faculty who take second author to mentor students Creation of research teams.

- Finally, creating an environment where people know they are supported and that their interests are essential to the group invites research teams to form and collaborate to develop.

- Inviting colleagues from my discipline and other disciplines to engage in research interests fosters multiple perspectives and requires input from various sources/ disciplines. I am open to an invitation to do so

- One strategy I have used is to create groups in which team members share mutual interests.

- Make sure that everyone understands the need and benefits of scholarly collaborative relationships.

Goal Setting. Goal setting is the primary step in keeping the group accountable. A good leader can control themselves and the others responsible (liable) for their actions. Knowing the expectations and outcomes is also a quick way to establish trust within the team. They are often meeting, setting deadlines, and modeling perseverance.

- It is important to set short-term goals to keep the momentum. Leaders have to be aware of varying demands placed on collaborators and form a mixture of achievable goals that are short-term and medium. Frequent check-ins help make sure collaborators are focused on achieving their goals.

- I periodically revisit the purpose of our organization to ensure that we are moving towards mutually shared goals. 
- Set those goals and make sure there are accountability mechanisms (e.g., mentoring and routine group meetings to discuss progress or lack thereof).

- One of the things I've come to terms with over the past few years is the following statement: "This is not a love affair; this is business." This quote was shared with me from a high-profile Carnegie Mellon alum when he provided career strategies on success for people of color. Thus, to maintain collegial relationships while conducting/navigating scholarship (research) business, I have to discern the working relationships to determine whether I focus only on the relationship's business aspect or integrate personal and business. If I focus on the business aspect, I remind myself that it is not a love affair; I can be collegial to get the job/task done.

Management. Management is the coordinating and the administration of tasks to achieve a goal. Standard components of management in different arenas (inclusive of academic scholarship collaboration) include selection, supervision, motivation, and evaluation of staff, scheduling, and workflow planning. I am careful about whom I collaborate with on research. I typically collaborate with individuals who can handle the ebb and flow of research and not take things personally.

- I try to compromise with authors based on the amount of work they contributed. If there is a prominent person in the profession, I might see if everyone is good with their name headlining. It also depends on the journal. In tenure and promotion processes (depending on the university), the order does not matter if you can showcase your contribution by percentages.

- It is vital that each team member "buy into" the project and support the work with their investment. When each person believes their contributions matter and invested in making these contributions, truly successful scholarly collaborations are possible. It is essential that the team members share positive energy (dreams/visions/ideas), and engage in honest discussions when they feel stuck, immobilized, or lack creativity.

\subsubsection{Institutional Research Capacity (Category 2)}

University capacity refers to the institution's ability to enable individuals and departments to undertake publications in peer-reviewed journals and successful grant submissions (Leitch, 2009). Research capacity also focuses on the end-user to be engaged and understand how to draw on research to inform future research priorities. Several sub-categories (Faculty resources and Conference engagement) support the tenets of a strong university research capacity. These identified areas create environments so that scholarship collaboration may occur.

Faculty Resources. All institutions, for the most part, offer some sort of support to their faculty. Notably, the support provided is not standardized; support is dependent on its mission and available financial assets. Research intensive institutions tend to have expectations and resources allocated for the creation and sustenance of scholarship.

- Our institution provides competitive scholarships for collaborative research, IRB support, research forums, and brown bags, and our college has a division dedicated to supporting faculty research agendas.

- Training and workshops see grants for transdisciplinary research collaborations.

- The organization currently has research and learning centers to support scholarly collaboration.

- Financial support, the release of time, IRB, and writing support workshops are available.

- All research-active faculty have GRA (graduate research assistant) support; we have methodological research support through our library services; we have technology supporting collaboration.

- Fosters collaboration, particularly mentoring with junior faculty, students, and recent alumni

- My institution provides the tools to us without cost. We can upgrade to maximize our capacity by utilizing our professional development funds. For example, my institution provides Dropbox to us for free; however, I pay $\$ 100$ per year to upgrade Dropbox because I keep many documents stored. We are provided a Zoom account that we can access through our department.

None of the participants mentioned teaching or work-life balance support as a necessary resource for successful scholarship collaboration. The teaching institutions offered education and classroom tools, such as instruction endowments, technology, coaching, and instructional help (Brooks, M, Alson, G., Townsend, C., \& Bryan, M., 2017).

Conference Engagement. Regular conference attendance has several professional benefits. Conferences offer the opportunity to meet like-minded people and industry peers. Conferences also bring together people from all different geographical areas who share a common discipline or field. The meetings offer professional development (continuing education units) and experienced leaders' chance to disseminate and share their work. Conferences, moreover, are 
incubators for generating new research/scholarship ideas.

- I attend conferences regularly and discuss research interests with colleagues. I have served on several research-oriented panels and have had graduate students, and new professionals seek me out for mentorship and consultation.

- I create opportunities at conferences and informal professional gatherings to brainstorm about potential ideas. Every discussion is an opportunity to collaborate on a project.

\subsubsection{Communication (Category 3)}

Communication is vital in most jobs and goal-oriented tasks. Professionals will likely need to communicate with people on the job, whether they are clients, customers, colleagues, employers, or vendors. Likewise, the researchers realize communication is often a subset of leadership (Austin, \& Baldwin, 1992; Austin, Hair, \& Fullerton, 2012) and could have appeared under an earlier heading; the data was strong enough to support it as a stand-alone category.

Openness: Effective communication requires openness, especially when collaborating. The ability to receive suggestions and changes as well as produce intentional conversations about collective goals. This type of open dialogue can go a long way in each member understanding and accepting individual expectations. Counselors require an account of openness for successful client outcomes. In collaborative writing, this same type of technique can indicate achieving buy-in from all collaborative partners.

- These are all lessons I have learned over 20+ years of doing research and collaborating, and I was not as open and communicative at the beginning of my career. I have learned that the best way to collaborate and maintain healthy relationships is to be transparent, honest, and flexible.

- Clear written expectations on roles, responsibilities, timelines, and deadlines.

- Communicating the expected outcomes and the roles each person will play in achieving the overarching goal(s).

- Through open communication, one can create an environment that maximizes collaborative potentials.

- Finally, it is vital to engage in honest communication regarding goals, expectations, strengths, challenges, and timelines.

- Being deliberate in communications related to expectations, timelines, and other aspects of the scholarly project. Seeking the participants' needs is essential to ensure the project's buy-in, the expectations that each person on the team has of other members.

- By being open to working with a variety of individuals who bring various skills to the table. Recognizing each individual's strengths and interests brings and explores ways to maximize those skills and interests to achieve common goals.

- I believe in the Covey techniques (The 7 Habits of Highly Effective People) and sports team concepts. First, it is crucial, to begin with, an end in mind. It is developing a vision and mission for collaborative scholarship relationships. It is also essential to learn the values and beliefs of the leaders and team members involved with the project — conflict resolution skills (conflict is inevitable); Compassion and empathy, and Commitment to the process.

- Being connected to the professional interests of our colleagues and inquiring about their interests helps support the culture.

Clarity Around Roles. Transparency is the clearness or lucidity about perception or understanding; freedom from indistinctness or ambiguity (Miriam-Webster, 2020). In collaborative writing, the ability to utilize this definition in explaining roles in the process can keep everyone honest, organized, and intentional in the work they produce towards the desired goal. With clarity around roles, contributors are free from the confusion that could hinder or impede progress to the desired outcome. Clarity around roles can also formulate ground rules, authorship, deadlines, and highlight each contributor's strengths. Responsibilities and timelines also help everyone stay organized, while sufficient flexibility allows the researchers to access their creativity and think through the implications and research write-up.

- Clear and transparent communication (e.g., expectations of contributors, timelines, and authorship order) Scaffolding expectations (e.g., not giving a junior collaborator a more complex task than they can handle) Leader enthusiasm and organization

- Clear written timelines and deliverable expectations with consequences (e.g., lose authorship or authorship number, removed from the team) Similar work ethic (e.g., the team who believes faculty should come to work and work 40 hours) 
- Communication, conflict resolution skills, compassion, commitment, patience, self-awareness of strengths and challenges (limitations), humbleness, TIME, mental performance

- After a very traumatic experience with a co-authors team with a book chapter, I now make sure this is a discussion upfront before roles and tasks are assigned.

Constant (ongoing) communication. Communication is paramount to any project, but especially to leadership positions. Knowing how to communicate thoughtfully and intelligently and listen intently and actively, keeping both in balance, will lead to success.

- I think open and transparent communication also fosters collegial relationships.

- I try to stay in close communication with collaborative colleagues. It is essential to regularly update each other to ensure everyone is on the same page and progress as expected. Sometimes conversations are necessary to keep colleagues moving along. It is essential to be honest in the communications, especially related to following ground rules set at the beginning of the process. Recognize that sometimes you have to re-group and make adjustments to the original collaborative plan. Communication and honest feedback are the keys to successfully maintaining collegial relationships.

- Collegial relationships are best maintained through honest and open communication. While the conversations that occur may be difficult, it is best to approach each colleague with the dignity and respect of a natural set of talks. In the end, most will appreciate and value that stance.

- I think open and transparent communication also fosters collegial relationships. When I am working with doctoral students, there will be times when they will get frustrated with the project and frustrated with, and that is just a part of the process. What I ask is when that happens, they come to discuss it with me.

\section{Discussions}

The results of this study directly connects to counselor educators who work in academic settings. Teamwork, newer technologies, and limited resources encourage such partnerships (Austin \& Baldwin, 1992). Moreover, it is well-documented that faculty experience feelings of being overwhelmed by competing demands on their time to fulfill university missions (Cumbie et al., 2005; Feldman \& Acord, 2002; Langston et al., 1999; Mundt, 2001). Often, faculty scholarship is pursued and accomplished on personal time (Feldman \& Acord).

Studies investigating such cause and their remediation included Jacobs and Winslow (2004), who suggested two explanatory models for faculty tendencies to work long hours. In the self-imposed or optimistic model, faculty chose to work long hours because of their love and "devotion to their work" (p. 108). In the second model, structural constraints or pessimistic views suggested that job requirements demand long hours at the expense of work satisfaction, family life, and, at times, physical and mental health. A negative association was found between long hours, job satisfaction, and family relations. These findings were valid, particularly in academic settings with increased expectations to use technology to produce even more work output and publish to retain their faculty positions. Academics are encouraged to find ways to establish boundaries against higher education's infinite needs in the interest of personal health and well-being. Collaborative scholarship probably speaks to these needs by sharing work duties, decreasing the number of single and group hours required to execute tasks, which points to an uptick in job satisfaction (Thompson, Galbraith \& Pedro, 2010).

The counseling professional governing body and higher education administrators may apply findings from this study to the scope and practice of being a counselor educator academician. The researchers believe that counseling requires intentional redefining of research approaches and methods regarding our relational, humanistic, and constructivist philosophies. These pillars define the counseling profession and distinguish us from other helping groups. An overhaul would require us to acknowledge relationships as a defining and constructive component of knowledge development and values around social justice and transformational practices. Our research may not look like others.

\section{Limitations and Considerations for Future Research}

There are some limitations of the study in need of acknowledgement. One limitation was the implementation of qualitative content analysis. The researchers experienced difficulties getting the participants to respond to the repeated and revised interview questions. As the study continued, participants required additional motivation and multiple reminders to complete question prompts. Future researchers who wish to continue this type of work may want to consider structured communication methods with substantial participants' commitments - the Delphi method forecasts (decisions) based on a panel of experts' opinions may be a possible consideration.

The second limitation deals with the profession's efforts to establish a greater professional counselor identity within our research approach and address how we see evidence-based practice and how we see outcome research, benefiting our humanistic, developmental, strength-based perspective. Achieving balance in academic life is (still) a goal to be realized 
by most faculty. Moreover, achieving balance requires a different way of thinking about scholarship, in general. Building on Boyer's (1990) model of scholarship, many counselor education authors and researchers may want to consider redefining, and therefore the re-evaluation, of scholarship to encompass more than just the traditional products of research (Sherwen, 1998; Sullivan, 1996; Ward, 2008). While scholarly works frequently are defined as grant submissions and manuscripts, the products of mentoring and collegial faculty discourse are just as crucial to scholarship, albeit less tangible (Young, 1984). Perhaps future studies can consider other ways of measuring the benefits of collaborative work.

\section{References}

Allen, J. L., Huggins-Hoyt, K. Y., Holosko, M. J., \& Briggs, H. E. (2017). African American social work faculty: Overcoming existing barriers and achieving research productivity. Research on Social Work Practice, 28(3), 309-319. https://doi.org/10.1177/1049731517701578

Austin, A. E., \& Baldwin, R. G. (1992). Faculty collaboration: Enhancing the quality of scholarship and teaching (ED347958). ERIC, pp. 1-6. https://files.eric.ed.gov/fulltext/ED347958.pdf

Austin, M. A., Hair, M. S., \& Fullerton, S. M. (2012). Research guidelines in the era of large-scale collaborations: An analysis of genome-wide association study consortia. American Journal of Epidemiology, 175(9), 962-969. https://doi.org/10.1093/aje/kwr441

Ayoola, A. B., Adams, Y. J., Kamp, K. J., Zandee, G. L., Feenstra, C., \& Doornbos, M. M. (2017). Promoting the Future of Nursing by Increasing Zest for Research in Undergraduate Nursing Students. Journal of Professional Nursing, 33(2), 126-132. https://doi.org/10.1016/j.profnurs.2016.08.011

Bozeman, B., \& Youtie, J. (2016). Trouble in Paradise: Problems in Academic Research Co-authoring. Science and Engineering Ethics, 22(6), 1717-1743. https://doi.org/10.1007/s11948-015-9722-5

Brooks, M., Alston, G., Townsend, C., \& Bryan, M. (2017). Creating a Healthy Classroom Environment in Multicultural Counseling Courses. Journal of Human Services: Training, Research, and Practice, 2, 1-24.

Brooks, M., Wolfgang, J., Adams, J., Armstrong, N., \& Cassidy, R. (2020). Using rap music to better understand African American experiences. Journal of Creativity in Mental Health, 1-17. https://doi.org/10.1080/15401383.2020.1732251

Carter, N., Bryant-Lukosius, D., DiCenso, A., Blythe, J., \& Neville, A. J. (2014). The use of triangulation in qualitative research. Oncology nursing forum, 41(5), 545-547. https://doi.org/10.1188/14.ONF.545-547

Creswell, J. W., \& Guetterman, T. C. (2019). Educational research: Planning, conducting, and evaluating quantitative and qualitative research (6th ed.). Upper Saddle River, NJ: Pearson Education, Inc.

Crowe, S., Cresswell, K., Robertson, A., Huby, G., Avery, A., \& Sheikh, A. (2011). The case study approach. BMC Medical Research Methodology, 11(1), 100. https://doi.org/10.1186/1471-2288-11-100

Driscoll, L. G., Parkes, K. A., Tilley-Lubbs, G. A., Brill, J. M., \& Pitts Bannister, V. R. (2009). Navigating the lonely sea: Peer mentoring and collaboration among aspiring women scholars. Mentoring \& Tutoring: Partnership in Learning, 17(1), 5-21. https://doi.org/10.1080/13611260802699532

Goldstein, D. G., \& Gigerenzer, G. (2002). Models of ecological rationality: The recognition heuristic. Psychological Review, 109(1), 75-90. https://doi.org/10.1037//0033-295X.109.1.75

Holstein, J. A., \& Gubrium, J. F. (2012). Varieties of narrative analysis. Los Angeles, CA: Sage Publications.

Hsieh, H. F., \& Shannon, S. E. (2005). Three approaches to qualitative content analysis. Qualitative Health Research, 15(9), 1277-1288. https://doi.org/10.1177/1049732305276687

Katz, J. S., \& Martin, B. R. (1997). What is research collaboration? Research Policy, 26(1), 1-18. https://doi.org/10.1016/S0048-7333(96)00917-1

Leitch, R. (2009). Harnessing the slipstream: Building educational research capacity in Northern Ireland. Size matters. Journal of Education for Teaching, 35(4), 355-371. https://doi.org/10.1080/02607470903220422

McConnell, D. (2016). Narrative self-constitution and vulnerability to co-authoring. Theoretical Medicine and Bioethics, 37(1), 29-43. https://doi.org/10.1007/s11017-016-9356-x

Paetow, G., Zaver, F., Gottlieb, M., Chan, T. M., Lin, M., \& Gisondi, M. A. (2018). Online mastermind groups: A non-hierarchical mentorship model for professional development. Cureus, 10(7), 1-8. https://doi.org/10.7759/cureus.3013

Patel, H., Pettitt, M., \& Wilson, J. R. (2012). Factors of collaborative working: A framework for a collaboration model. Applied Ergonomics, 43(1), 1-26. https://doi.org/10.1016/j.apergo.2011.04.009 
Saldaña, J. (2016). The coding manual for qualitative researchers. (3rd ed.). London, UK: Sage Publications.

Thompson, C. J., Galbraith, M. E., \& Pedro, L. W. (2010). Building collaborative scholarship in an academic nursing community. International Journal of Nursing Education Scholarship, 7(1). https://doi.org/10.2202/1548-923X.1922

Tsatsou, P. (2016). Digital technologies in the research process: Lessons from the digital research community in the UK. Computers in Human Behavior, 61, 597-608. https://doi.org/10.1016/j.chb.2016.03.053

Tufford, L., \& Newman, P. (2010). Bracketing in qualitative research. Qualitative Social Work, 11, 1-17. https://doi.org/10.1177/1473325010368316

Vaismoradi, M., Jones, J., Turunen, H., \& Snelgrove, S. (2016). Theme development in qualitative content analysis and thematic analysis. Journal of Nursing Education and Practice, 6(5), 100-110. https://doi.org/10.5430/jnep.v6n5p100

Vereen, L. G., Hill, N. R., Sosa, G. A., \& Kress, V. (2014). The synonymic nature of professional counseling and humanism: Presuppositions that guide our identities. The Journal of Humanistic Counseling, 53(3), $191-202$. https://doi.org/10.1002/j.2161-1939.2014.00056.x

World Health Organization. (2010). Framework for action on interprofessional education \& collaborative practice (No. WHO/HRH/HPN/10.3). Geneva, Switzerland: World Health Organization. See Retrieved from http://whqlibdoc.who.int/hq/2010/WHO_HRH_HPN_10.3_eng.pdf

Yin, R. K. (2018). Case study research and applications: Design and methods (6th ed.). Thousand Oaks, CA: Sage Publications.

\section{Copyrights}

Copyright for this article is retained by the author(s), with first publication rights granted to the journal.

This is an open-access article distributed under the terms and conditions of the Creative Commons Attribution license which permits unrestricted use, distribution, and reproduction in any medium, provided the original work is properly cited. 